\title{
Geographic variations, cloning, and functional analyses of the venom acidic phospholipases $\mathrm{A}_{2}$ of Crotalus viridis viridis ${ }^{\text {is }}$
}

\author{
Inn-Ho Tsai, ${ }^{\mathrm{a}, *}$ Ying-Ming Wang, ${ }^{\mathrm{a}}$ Yi-Hsuan Chen, ${ }^{\mathrm{a}}$ and Anthony T. Tu ${ }^{\mathrm{b}}$ \\ a Institute of Biological Chemistry, Academia Sinica, and Institute of Biochemical Sciences, National Taiwan University, Taipei, Taiwan \\ ${ }^{\mathrm{b}}$ Department of Biochemistry, Colorado State University, Fort Collins, Colorado 80523, USA
}

Received 6 December 2002, and in revised form 26 December 2002

\begin{abstract}
Geographic venom samples of Crotalus viridis viridis were obtained from South Dakota, Wyoming, Colorado, Oklahoma, Texas, New Mexico, and Arizona. From these samples, the phospholipases $\mathrm{A}_{2}\left(\mathrm{PLA}_{2} \mathrm{~s}\right)$ were purified and their N-terminal sequences, precise masses, and in vitro enzymatic activities were determined. We purified two to four distinct acidic PLA $\mathrm{S}_{2}$ from each sample; some of them displayed different inhibition specificities toward mammalian platelets. One of the acidic PLA ${ }_{2} \mathrm{~S}$ induced edema, but had no anti-platelet activity. There was also a common basic PLA $\mathrm{P}_{2}$ myotoxin in all the samples. We have cloned five acidic PLA 2 and several hybrid-like nonexpressing $\mathrm{PLA}_{2} \mathrm{~s}$. Molecular masses and N-terminal sequences of the purified PLA $\mathrm{A}_{2} \mathrm{~S}$ were matched with

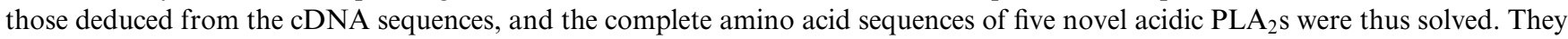
share $78 \%$ or greater sequence identity, and a cladogram based on the sequences of many venom acidic PLA 2 s of New World pit vipers revealed at least two subtypes. The results contribute to a better understanding of the ecogenetic adaptation of rattlesnakes and the structure-activity relationships and evolution of the acidic $\mathrm{PLA}_{2} \mathrm{~s}$ in pit viper venom.
\end{abstract}

(C) 2003 Elsevier Science (USA). All rights reserved.

Keywords: Crotalus v. viridis; Snake venom; Phospholipase $\mathrm{A}_{2}$; Cloning; Complete sequence; Geographic variation; Platelet aggregation; Edema

Phospholipases $\mathrm{A}_{2}\left(\mathrm{PLA}_{2} \mathrm{~s}\right)^{1}$ are present in most, if not all, pit viper venoms. This secreted enzyme family is relatively stable, easy to be purified, and without posttranslational modifications. The amino acid sequences of about $200 \mathrm{PLA}_{2}$ s from snake venom have been determined and many of their 3-D structures were determined $[1,2]$. The venom PLA $_{2}$ s have evolved into several functional subtypes, which may play different biological roles such as platelet aggregation inhibitor, neurotoxin, anticoagulant, or myotoxin [3,4]. The acidic $\mathrm{PLA}_{2} \mathrm{~S}$

\footnotetext{
Sequence data from this article have been deposited with the EMBL Data Library under Accession Nos. AF403134-AF403137 for Cvv-E6a, f, g, and $\mathrm{h}$ and Accession Nos. AY120875-AY120877 for Cvv-E6e, d, and b, respectively.

${ }^{*}$ Corresponding author. Fax: +886-2-2363-5038.

E-mail address: bc201@gate.sinica.edu.tw (I.-H. Tsai).

${ }^{1}$ Abbreviations used: Cvv, Crotalus viridis viridis; dPPC, L-dipamitoylglycerophosphatidylcholine; $\mathrm{PLA}_{2}$, phospholipase $\mathrm{A}_{2}$; ESIMS, electrospray ionization-mass spectrometry; PRP, platelet-rich plasma.
}

usually show strong hydrolytic activity and may inhibit the aggregation of platelets $[5,6]$.

Prairie rattlesnakes (Crotalus viridis viridis, abbreviated Cvv) inhabit the region east of the Rocky Mountains in the United States, from southern Canada to northern Mexico [7]. Its microhabitats, including prairie, cropland, grassland, and desert, are diverse. Some of the venom components have been purified and studied, including a kallikrein-like protease [8], the 5-kDa myotoxins [9], and a basic and myotoxic PLA 2 [10], but not the acidic $\mathrm{PLA}_{2} \mathrm{~s}$. The intraspecies variations of its venoms collected in Texas and New Mexico have been studied by electrophoresis and immunochemistry [11]. Recently, the phylogenetic analyses based on mitochondrial DNA sequences revealed the taxa variations of $\mathrm{Cvv}$ from different localities [12].

In this study, the intraspecies diversity of venom composition and its significance are investigated at the molecular level using $\mathrm{PLA}_{2} \mathrm{~S}$ as the window. We analyzed the venom of $\mathrm{Cvv}$ using the samples from seven localities of its range. The acidic venom $\mathrm{PLA}_{2} \mathrm{~s}$ were 
purified, cloned, and sequenced. Their functional activities, including inhibition of the aggregation of platelets from several species, were compared. Moreover, we

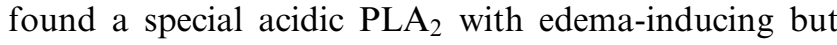
not anti-platelet activity. The amino acid sequences of these $\mathrm{PLA}_{2} \mathrm{~S}$ were compared to discuss the structureactivity relationships, and a phylogeny tree based on these sequences was built to decipher their structural or evolutionary relationships.

\section{Materials and methods}

Venoms and other materials. Lyophilized samples of $\mathrm{Cvv}$ venom from Wyoming and South Dakota were purchased from Miami Serpentarium Laboratory and Kentucky Reptile Zoo, respectively. Professor Steve P. Mackessy (University of Northern Colorado, Greeley, CO, USA) supplied the Cvv venom from Colorado as a gift, and Professor Eppie Rael (University of Texas, El Paso, TX, USA) generously donated the Cvv venoms from Texas, New Mexico, and western Oklahoma. A live specimen ( $\mathrm{Cvv}$ No.2) of south Arizona origin was purchased from Glades Herps, Inc. (Fort Myers, FL, USA). Its venom was obtained 2 days before the venom glands were taken and the snake was sacrificed. The mRNA extraction and the cDNA synthesis kits were from Stratagene. Modification and restriction enzymes were purchased from Promega. Synthetic dipalmitoyl phosphatidylcholine was purchased from Avanti Polar Lipids (Alabaster, AL, USA). Other chemicals were of reagent grade.

Purification and assay of PLA $A_{2} S$. Dissolved Cvv venom (3-15 mg) was fractionated on a FPLC system with a column of Superdex G-75 (HR10/30, Pharmacia) in $0.1 \mathrm{M}$ ammonium acetate $(\mathrm{pH} 4.2)$ at room temperature. The fractions corresponding to $12 \pm 2$ and $26 \pm 2 \mathrm{kDa}$ were pooled separately and lyophilized. These PLA $_{2-}$ containing fractions were further fractionated by reversed-phase HPLC using a column of silica gel (Vydac $\left.\mathrm{C}_{8}, \quad 14 \times 250 \mathrm{~mm}, \quad 10 \mu \mathrm{m}\right)$ equilibrated with $0.07 \%$ aqueous trifluoroacetic acid (solvent $\mathrm{A}$ ) and eluted with a $25-45 \%$ linear gradient of $\mathrm{CH}_{3} \mathrm{CN}$ containing $0.07 \%$ trifluoroacetic acid (solvent $\mathrm{B}$ ). The purified $\mathrm{PLA}_{2} \mathrm{~S}$ were dried in a vacuum-centrifuge device (Labconco, USA).

The dye-staining method of Bradford was used for quantification of the purified $\mathrm{PLA}_{2} \mathrm{~s}$. Bovine serum albumin $\left(1 \mathrm{mg} / \mathrm{ml}, A_{280}=0.56\right)$ was used to prepare the standard curve. $\mathrm{PLA}_{2}$ activity was measured by the $\mathrm{pH}$ stat titration method [13] using the micellar substrates of L-dipalmitoylglycerophosphatidylcholine (dPPC; $3 \mathrm{mM}$ ) with a pH-stat apparatus (RTS 822; Radiometer, Denmark). The reaction rate was corrected for the nonenzymatic spontaneous rate.

Amino acid sequences and molecular mass. The $\mathrm{N}$ terminal sequences of purified $\mathrm{PLA}_{2} \mathrm{~S}$ were determined by an automated amino acid sequencer (Model 477A; PE-Applied Biosystems). Molecular weights of the $\mathrm{PLA}_{2} \mathrm{~S}$ were determined by ESI-MS on a Sciex mass analyzer (API100; Perkin-Elmer) using about $2 \mu \mathrm{g}$ of the enzyme dissolved in $20 \mu 1$ of $0.1 \%$ acetic acid with $50 \%(\mathrm{v} / \mathrm{v}) \mathrm{CH}_{3} \mathrm{CN}$.

Cloning and sequencing. The cDNA library of Cvv venom glands from a Texas specimen (Cvv No.1) had been previously constructed [14]. For Cvv No.2 from Arizona, the venom gland mRNA and the cDNA were prepared as before $[13,15]$. In order to amplify and clone $\mathrm{PLA}_{2} \mathrm{~s}, \mathrm{PCR}$ [16] was conducted using SuperTaq DNA polymerase with a pair of mixed-base oligonucleotide primers (primer 1, TCTGGATTG/CAGGAGGATGA GG, and primer 2, GCCTGCAGA/GACTTAGCA), which were designed according to the highly conserved cDNA regions of the group II PLA $\mathrm{P}_{2}$ s from snake venoms $[13,17]$. Fragments of $0.4 \mathrm{~kb}$ were specifically amplified by PCR as shown by electrophoresis of the products on a $1 \%$ agarose gel.

After being treated with polynucleotide kinase, the amplified DNA was inserted into the pGEM-T-Easy vector (Promega Biotech, Madison, WI, USA). Then, it was transformed into Escherichia coli strain JM109. White transformants were picked up and cDNA clones were selected. The DNA sequencing system Model 373A and the Taq Dye-Deoxy terminator-cycle sequencing kit (PE-Applied Biosystems) were used to determine the cDNA sequences by the dideoxynucleotide method [18].

Platelet aggregation and edema. Blood was collected from a healthy human donor, rabbit, and guinea pig, in the presence of $3.8 \%$ sodium citrate $(9: 1, \mathrm{v} / \mathrm{v})$. The blood was centrifuged at $130 \mathrm{~g}$ for $15 \mathrm{~min}$ at room temperature to prepare platelet-rich plasma (PRP). Platelet aggregation was measured by an aggregometer (Payton, Model $600 \mathrm{~B}$, Canada) at $37^{\circ} \mathrm{C}$. Aliquots $(0.45 \mathrm{ml})$ of PRP were preincubated with the tested PLA $_{2}$ for $5 \mathrm{~min}$ in a siliconized glass cuvette under constant stirring. The aggregation was initiated by the addition of ADP to a final concentration of $10 \mu \mathrm{M}$ and followed for 5-10 min. The dose-dependent inhibition of the ADP-induced aggregation of PRP by the purified PLA $\mathrm{P}_{2}$ was investigated.

The edema-inducing activity of the purified $\mathrm{PLA}_{2}$ on the hind paw of Wistar rats (female, weight about $250 \mathrm{~g}$ ) was measured as previously described [15]. A plethysmometer (Type 7150; Ugo Basile) monitored the time course of the swelling.

Phylogenetic analysis of the acidic venom PLA $A_{2} \mathrm{~S}$. The phylogenetic tree was constructed based on amino acid sequences of the venom acidic $\mathrm{PLA}_{2} \mathrm{~s}$ so far available for the New World pit vipers. Multiple alignments of the sequences were based on the program PILEUP. Neighbor-joining method was used in the program PHYLIP to build the tree [19]. Confidence of the linkage at each node was estimated by bootstrap analyses of 1000 replicas [20]. 


\section{Results}

\section{Purification and characterization of venom PLA $A_{2} \mathrm{~S}$}

Separation of dimeric and monomeric $\mathrm{PLA}_{2} \mathrm{~S}$ from the $\mathrm{Cvv}$ venom was achieved by gel-filtration (Fig. 1). From each of the $26-\mathrm{kDa}$ fractions, two or three acidic $\mathrm{PLA}_{2}$ isoforms were purified by reversed-phase HPLC (Fig. 2). Residue 6 in each of the acidic enzymes is Glu (i.e., E6), thus the $\mathrm{PLA}_{2} \mathrm{~s}$ are designated Cvv-E6a-f. Additionally, a single Asn6-containing basic PLA $2, \mathrm{Cvv}-\mathrm{N} 6$, was found in all the geographic samples. It was purified by HPLC from the $13-\mathrm{kDa}$ fraction of each sample (not shown). The molecular mass was determined by ESI-MS and then the enzymatic activities of potential $\mathrm{PLA}_{2} \mathrm{~s}$ were assayed. A total of seven distinct $\mathrm{PLA}_{2} \mathrm{~s}$ were identified from all the geographic samples, and their $\mathrm{N}$-terminal sequences were determined as shown in Table 1. Notably, the molecular weights for Cvv-E6b, Cvv-E6c, and Cvv-E6e are different although their N-terminal 23 residues are identical.

Among the seven geographic samples, only those from Arizona, New Mexico, and Texas are from individual snakes, the others are pooled venom samples. Their relative yields were calculated from the areas under the HPLC absorbance peaks (Table 2). Total content of the venom $\mathrm{PLA}_{2} \mathrm{~S}$ was usually $4-7 \%$ of the venom mass, except a higher content of $9.5 \%$ was found in the South Dakota sample. The content of Cvv-N6 apparently increased as the snakes' habitat moved farther to the South (Table 2). The N-terminal sequence of CvvN6 was consistent with that reported previously for the myotoxic PLA $\mathrm{P}_{2}$ of this venom [10].

\section{Cloning and sequence determination}

The venom gland cDNAs of Cvv No.1 and No.2 were used separately as templates in the PCR amplification of the $\mathrm{PLA}_{2} \mathrm{~s}$. After electrophoresis, the $0.4-\mathrm{kb}$ products were harvested from agarose gel. The cDNAs were cloned and sequenced by normal procedures [18] and their nucleotide sequences were checked twice. They all encoded a signal peptide of 16 amino acid residues followed by a PLA $\mathrm{P}_{2}$ domain of 122 residues. All the venom $\mathrm{PLA}_{2}$ clones reported herein were independently identified more than once.

Of about 90 clones of $\mathrm{PLA}_{2}$ selected from the cDNA library of Cvv No.1, about 50 encoded Cvv-E6f, the rest encoded Cvv-E6a and Cvv-N6. The majority of the 40 clones from cDNA of Cvv No.2 encoded Cvv-N6 and Cvv-E6e, 4 encoded Cvv-E6d, and only 1 encoded CvvE6b. However, Cvv-E6b was not isolated from the venom of Cvv No.2; it was isolated from the Colorado and Wyoming samples only (Table 2). We failed to clone Cvv-E6c, which was probably expressed only in the venom of $\mathrm{Cvv}$ from the northern range of its distribution (e.g., from South Dakota). The deduced amino acid sequences are aligned in Fig. 3 according to the commonly used numbering system [21]. The theoretical $\mathrm{p} I$ values of all 5 Cvv-E6 are between 4.9 and 5.6, while that of Cvv-N6 is 8.8 .
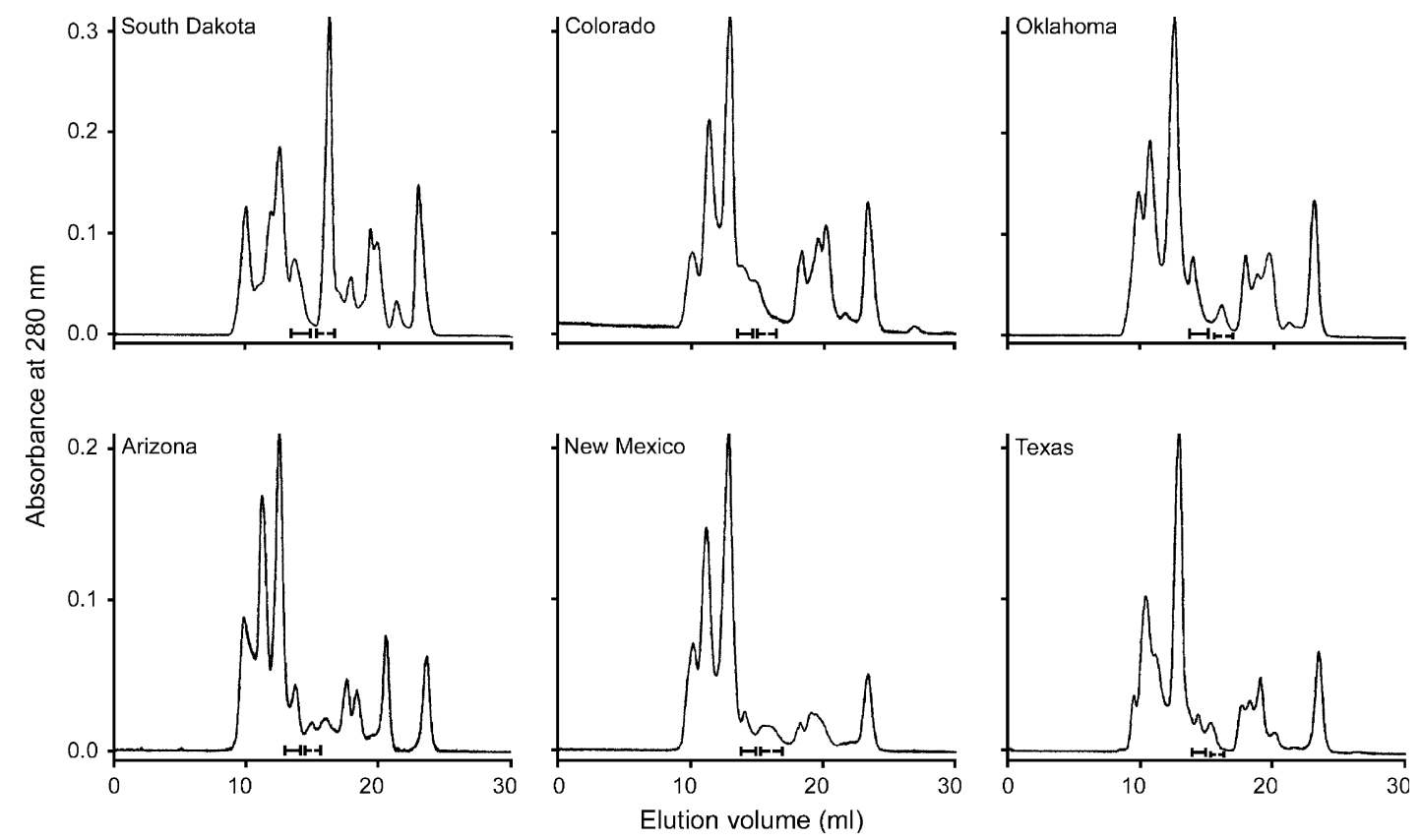

Fig. 1. Gel filtration of the crude venom of C. v. viridis. C. v. viridis venom samples collected from different states were loaded onto a Superdex G75 (HR10/30) column. Elution was carried out with the equilibration buffer, $0.1 \mathrm{~N}$ ammonium acetate ( $\mathrm{pH} 6.0$ ), at a flow rate of $1.0 \mathrm{ml} / \mathrm{min}$. Active fractions containing the dimeric (first bar) and the monomeric (second bar) $\mathrm{PLA}_{2} \mathrm{~s}$ were pooled, respectively. 

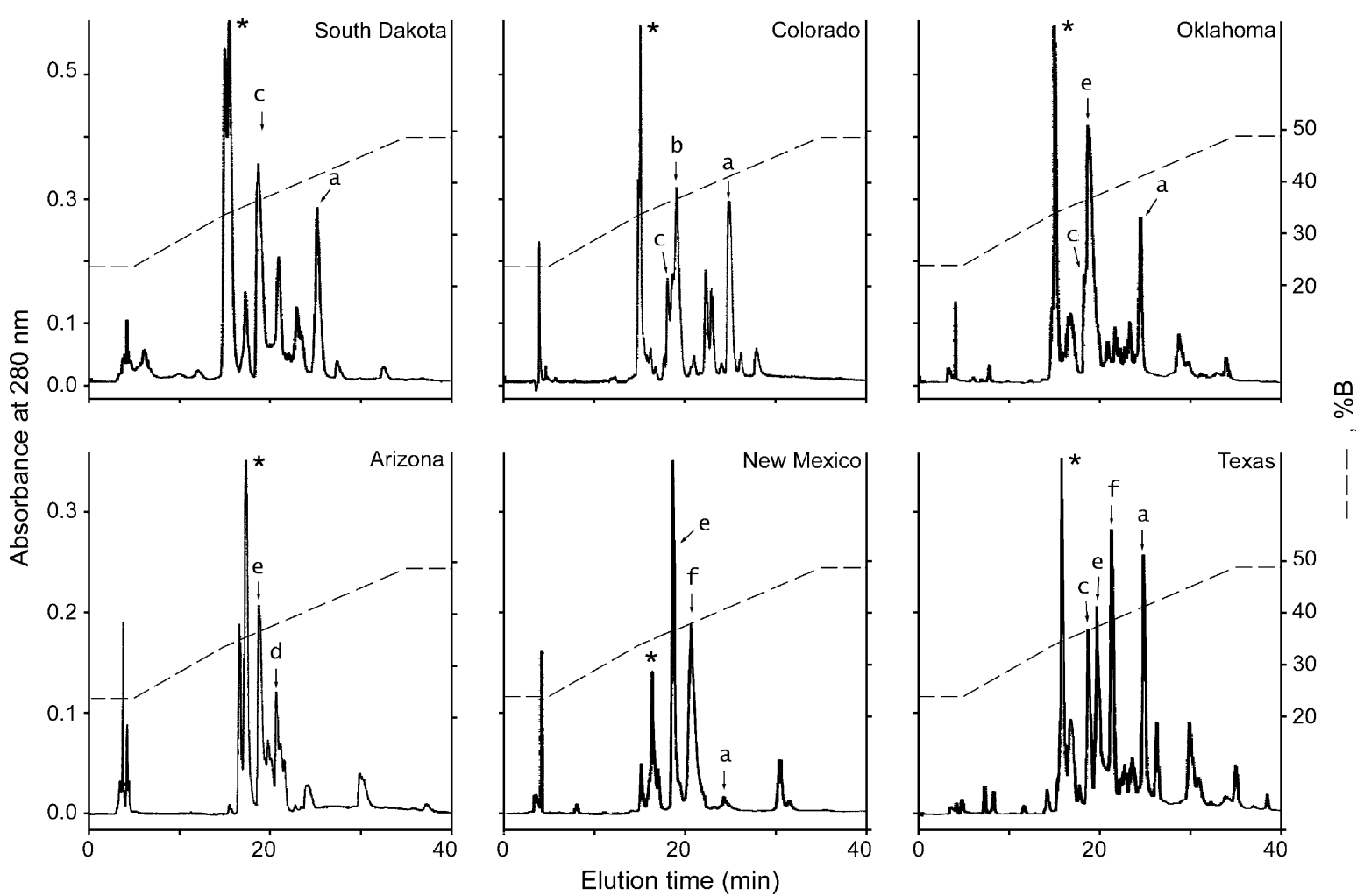

Fig. 2. Purification of the $\mathrm{PLA}_{2} \mathrm{~s}$ by reversed-phase HPLC. Lyophilized 26-kDa fractions from Fig. 1 were redissolved in solvent A and each was fractionated on a $\mathrm{C}_{8}$-Vydac HPLC column with a gradient of solvent B (dashed lines). All the purified PLA $\mathrm{A}_{2}$ s were confirmed by ESI-MS and enzymatic assay. The annotations are the same as those shown in Table 1. The peak for a serine protease (mass 24855) is marked with an asterisk.

Table 1

The elution conditions, molecular masses, and enzymatic activities of the acidic $\mathrm{PLA}_{2} \mathrm{~s}$ of Crotalus v. viridis venom

\begin{tabular}{|c|c|c|c|c|c|c|}
\hline \multirow[t]{2}{*}{ CVV-PLA } & \multirow[t]{2}{*}{ HPLC, $\% \mathrm{~B}$} & \multicolumn{2}{|l|}{ Mass } & \multicolumn{2}{|c|}{ Enzyme activities, ${ }^{\mathrm{a}} \mathrm{dPPC}$} & \multirow[t]{2}{*}{$\mathrm{N}$-terminal sequences ${ }^{\mathrm{b}}$} \\
\hline & & ESI-MS & Theoretical & +Deoxycholate & +Triton X-100 & \\
\hline E6a & 39 & $13467 \pm 1$ & 13467.2 & $351 \pm 16$ & $208 \pm 8$ & SLVQFETLIMKIAGRSGLLWYSA \\
\hline E6b & 36 & $13660 \pm 1$ & 13659.5 & $1129 \pm 20$ & $764 \pm 7$ & $\mathrm{~N} \cdots \mathrm{L} \cdots \mathrm{V} \cdot \mathrm{K} \cdots \mathrm{S} \cdots$ \\
\hline E6c & 35 & $13817 \pm 1$ & - & $840 \pm 6$ & $378 \pm 10$ & $\mathrm{~N} \cdots \cdots \mathrm{L} \cdots \mathrm{V} \cdot \mathrm{K} \cdots \mathrm{S} \cdots$ \\
\hline E6d & 37 & $13782 \pm 1$ & 13782.5 & $680 \pm 5$ & $345 \pm 10$ & $\cdots \cdots \mathrm{M} \cdots \mathrm{V} \cdot \mathrm{K} \cdots \mathrm{FS} \cdots$ \\
\hline E6e & 36 & $13633 \pm 1$ & 13633.5 & $1306 \pm 42$ & $678 \pm 29$ & $\mathrm{~N} \cdots \mathrm{L} \cdot \cdots \mathrm{V} \cdot \mathrm{K} \cdots \mathrm{S} \cdots$ \\
\hline E6f & 37 & $13876 \pm 1$ & 13875.7 & $518 \pm 25$ & $118 \pm 4$ & $\cdots \cdots \mathrm{MM} \cdot \mathrm{I} \cdot \mathrm{V} \cdot \mathrm{K} \cdots \mathrm{F} \cdot \mathrm{G} \cdot$ \\
\hline N6 & 33 & $14200 \pm 1$ & 14199.6 & $280 \pm 8$ & $88 \pm 4$ & $\mathrm{~N} \cdot \mathrm{L} \cdot \mathrm{NKM} \cdot \mathrm{KMMTKKNAFPF} \cdot \mathrm{TS}$ \\
\hline
\end{tabular}

${ }^{\mathrm{a}}$ Duplicate determination of the initial rate toward $3 \mathrm{mM} \mathrm{dPPC}$, in the presence of $10 \mathrm{mM} \mathrm{Ca}^{2+}$ at $37^{\circ} \mathrm{C}$. Values shown are means \pm error $(\mu \mathrm{mol} /$ $\mathrm{min} / \mu \mathrm{g}$ enzyme).

${ }^{\mathrm{b}}$ The N-terminal 23 residues are shown; those identical to the first line are marked with a dot.

In addition, we obtained five unusual PLA $_{2}$ clones, which did not match any of the purified PLA $\mathrm{P}_{2}$ (Fig. 4). These appeared to be the hybrid types, i.e., with the Nterminal and the C-terminal sequences derived, respectively, from different PLA variants found in the venom. The hybrid clones from individual specimens were different; PLA-g and h were from Cvv No.1 and PLA-i, j, and $\mathrm{k}$ were from Cvv No.2.

\section{Functional analyses of the acidic $P L A_{2} S$}

The inhibitions of the ADP-induced aggregation of the platelets from mammalian blood by the Cvv-E6 variants were analyzed. We found that Cvv-E6b, $\mathrm{d}$, and $\mathrm{f}$ showed very low anti-platelet activity toward platelet-rich plasma of human, rabbit (Fig. 5), or guinea pig (not shown), whereas Cvv-E6a, c, and e showed significant inhibition. Notably, Cvv-E6a was a more potent inhibitor than Cvv-E6e for the human and guinea pig platelets, but the relative potency was reversed in the case of rabbit platelets (Fig. 5).

In the search for possible functions for the Cvv-E6 variants without obvious anti-platelet activity, their edema-inducing activities were also tested. We found that Cvv-E6f effectively induced local swelling of the 
Table 2

Geographic variation in the venom $\mathrm{PLA}_{2} \mathrm{~S}$ of $C$. v. viridis

\begin{tabular}{|c|c|c|}
\hline Locality & CVV-PLA ${ }_{2} \mathrm{~S}$ & $\begin{array}{l}\text { Relative abundance } \\
(\%)^{\mathrm{a}}\end{array}$ \\
\hline \multirow[t]{3}{*}{ South Dakota } & E6a & 3.2 \\
\hline & E6c & 5.8 \\
\hline & N6 & 1.0 \\
\hline \multirow[t]{4}{*}{ Wyoming } & E6a & 1.5 \\
\hline & E6b & 0.6 \\
\hline & E6c & 1.4 \\
\hline & N6 & 1.1 \\
\hline \multirow[t]{4}{*}{ Colorado } & E6a & 0.7 \\
\hline & E6b & 3.5 \\
\hline & E6c & 0.4 \\
\hline & N6 & 1.5 \\
\hline \multirow[t]{4}{*}{ Western Oklahoma } & E6a & 0.8 \\
\hline & E6c & 0.4 \\
\hline & E6e & 1.4 \\
\hline & N6 & 2.1 \\
\hline \multirow[t]{5}{*}{ Texas } & E6a & 0.6 \\
\hline & E6c & 0.3 \\
\hline & E6f & 1.6 \\
\hline & E6e & 0.6 \\
\hline & N6 & 3.5 \\
\hline \multirow[t]{4}{*}{ New Mexico } & E6a & 0.1 \\
\hline & E6f & 1.3 \\
\hline & E6e & 1.6 \\
\hline & N6 & 2.2 \\
\hline \multirow[t]{4}{*}{ Southeastern Arizona } & E6a & 0 \\
\hline & E6d & 0.8 \\
\hline & E6e & 1.2 \\
\hline & N6 & 1.0 \\
\hline
\end{tabular}

${ }^{\mathrm{a}}$ The relative abundance was calculated based on the UV absorbance area under the PLA 2 peak in HPLC and calibrated with the total absorbance with respect to the crude venom (weight/weight).

hind paw while other acidic PLA $_{2}$ variants did not. The myotoxic Cvv-N6 elicited moderate edematous effect (Fig. 6).

\section{Phylogenetic tree of the venom acidic $P L A_{2} S$}

A cladogram was constructed to study the structural relationships between the venom acidic $\mathrm{PLA}_{2} \mathrm{~S}$ of $\mathrm{CrO}$ talus and those of other New World pit vipers. The protein sequence tree shown in Fig. 7 reveals an affinity among the Glu6-containing $\mathrm{PLA}_{2} \mathrm{~S}$ in the Crotalus venoms, with the robustness at most major nodes supported by bootstrap analysis. The tree also indicates that the acidic $\mathrm{PLA}_{2} \mathrm{~S}$ of $\mathrm{Cvv}$ venom may have evolved into two subtypes, one with anti-platelet activity, e.g., Cvv-E6a and the acidic venom $\mathrm{PLA}_{2}$ s from $C$. atrox and $C$. adamanteus, while the other may have other functions or specificities, e.g., Cvv-E6f becomes edema inducing (Fig. 6 ). The phylogenetic analysis using the cDNA sequences of these $\mathrm{PLA}_{2} \mathrm{~S}$ gave almost the same tree topology (not shown).

\section{Discussion}

The gel-filtration patterns of the Cvv venoms from different localities reveal more or less proportional differences (Fig. 1). These samples are rich in highmolecular-weight components $(>32 \mathrm{kDa})$, and their geographic variations may be attributed to venom proteins other than $\mathrm{PLA}_{2} \mathrm{~S}$ [11]. The South Dakota Cvv venom has relatively high content of the $8-$ to $9-\mathrm{kDa}$ polypeptides (Fig. 1), possibly disintegrins [22].

There are several $\mathrm{PLA}_{2}$ isoforms in the $\mathrm{Cvv}$ venom samples (Table 2). We identified the cDNA match for each of the $\mathrm{PLA}_{2}$ purified except Cvv-E6c (Fig. 3). The anti-platelet Cvv-E6a was present in all the $\mathrm{Cvv}$ venoms except the one collected from Arizona, in which another anti-platelet Cvv-E6e was found. In fact, a geographic North to South transition for the relative proportions of these $\mathrm{PLA}_{2} \mathrm{~S}$ was displayed, namely, Cvv-E6a and CvvE6c are decreasing, while Cvv-E6f, Cvv-E6e, and CvvN6 are increasing. Notably, the South Dakota sample

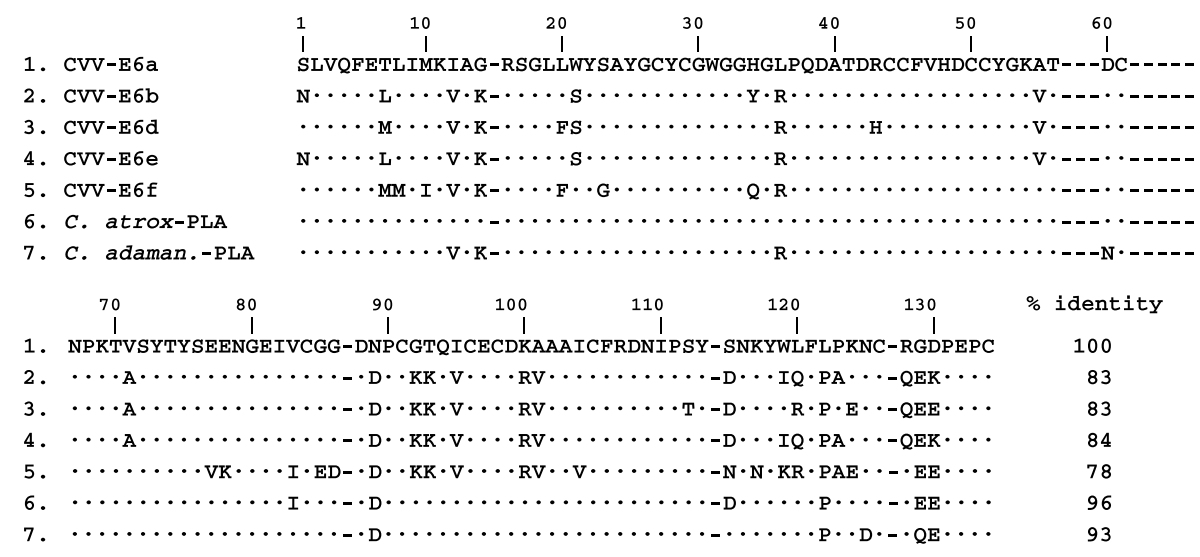

Fig. 3. Multiple alignments of the amino acid sequences of Cvv-E6 PLA $\mathrm{P}_{2}$. The amino acid sequences are deduced from the cDNA sequences and single-letter codes are used. The numbering system follows that of Renetseder et al. [21]. Residues identical to those in the top line are denoted with dots, gaps are marked with hyphens. The sequences of the venom $\mathrm{PLA}_{2} \mathrm{~s}$ of C. atrox and C. adamanteus are listed for comparison. 


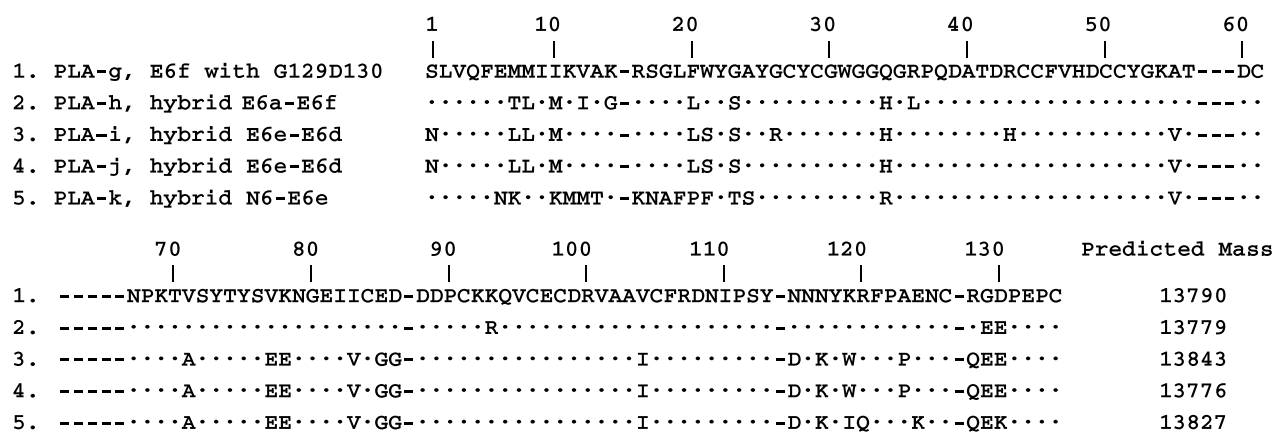

Fig. 4. Predicted amino acid sequences of the hybrid-like PLA 2 clones. The possible origins of their genes or structural features are denoted for each clone.

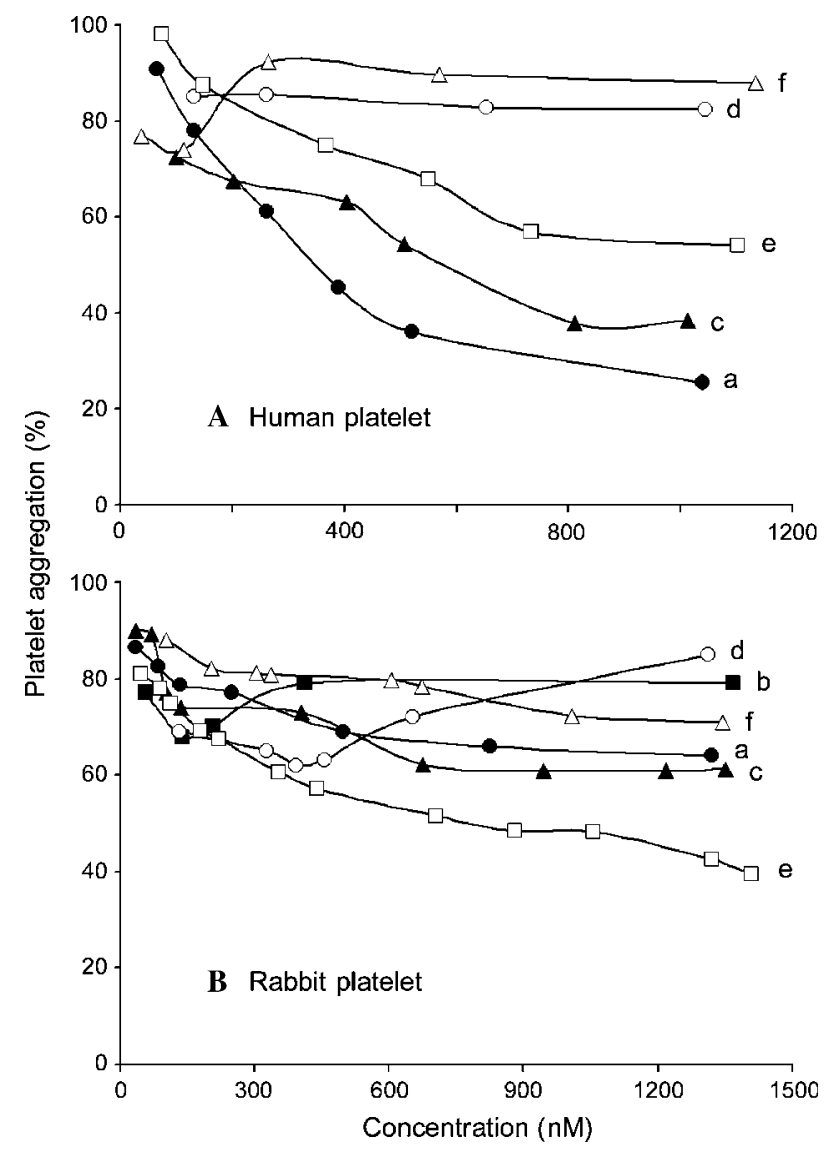

Fig. 5. Dose-dependent inhibition of platelet aggregation by Cvv-E6 $\mathrm{PLA}_{2} \mathrm{~s}$. The ADP-induced aggregation was studied at various Cvv-E6 concentrations with platelet-rich plasma of human and rabbit. The data points are the averages from two or three experiments.

contains only Cvv-E6c, while both Cvv-E6c and E6b are present in the pooled venom from Wyoming, which possibly represents a mixture of the South Dakota and the Colorado venoms. Thus, Cvv-E6c and E6b appear to be the markers for the $\mathrm{Cvv}$ venoms from their northern habitat, while Cvv-E6e and Cvv-E6f appear to be associated with those from the southern range, and Cvv-E6d is limited only to those from the most southwestern range (Arizona).

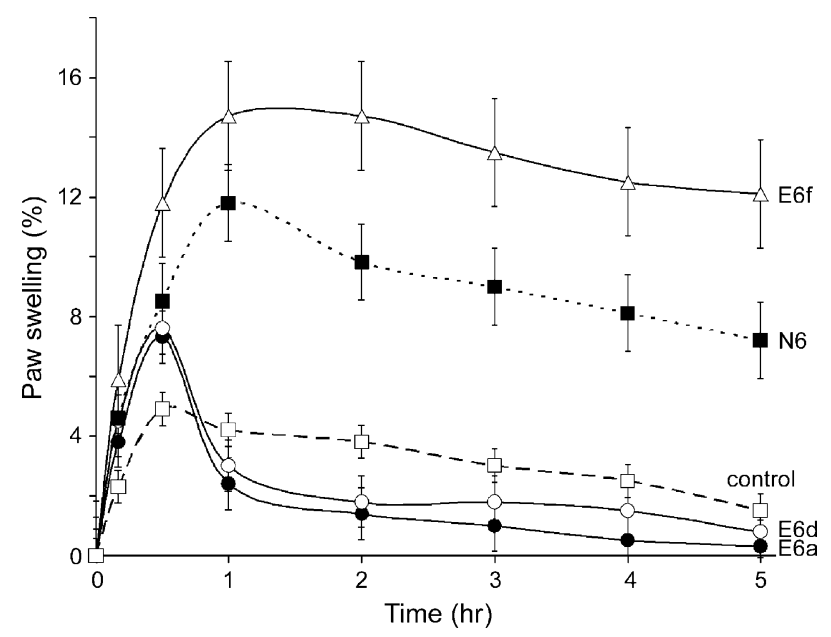

Fig. 6. Edema-inducing activities of some selected Cvv PLA 2 s. Rat paws were injected with $8 \mu \mathrm{g}$ of the purified venom PLA $_{2}$ in $100 \mu \mathrm{l}$ sterile saline $(0.9 \% \mathrm{NaCl})$. Saline solution was injected as the control. The volumes of the hind paws were measured by a plethysmometer. Experiments were done in duplicate, and data points are the average values with errors indicated.

We also found that the contents of Cvv-E6e varied significantly between the individual $\mathrm{Cvv}$ venoms collected from Texas and New Mexico (data not shown). This is consistent with the results from the venom studies using electrophoresis [11]. Moreover, previous phylogenetic analyses using the mitochondrial DNA sequences [12,23] showed that the Cvv samples from Nebraska and Montana were close to those from Wyoming and Colorado, while a sample from Bernalillo (New Mexico) was branched out from the rest of $\mathrm{Cvv}$ [23]. This and our finding that only the venom of Arizona Cvv contains Cvv-E6d (Table 2) suggest the presence of a distinct population of $\mathrm{Cvv}$ in its most southwestern habitat.

Based on the cDNA sequence data, we found that all the signal peptides of the Cvv-E6 isoforms and that of the acidic $\mathrm{PLA}_{2}$ of $C$. atrox (GenBank Accession No. AF269131) were identical, but differed from the signal peptide of Cvv-N6 $\mathrm{PLA}_{2}$ by three amino acid substitutions (not shown). Moreover, the amino acid sequence 
of Cvv-E6a is $>93 \%$ similar to those of the acidic PLA $_{2} \mathrm{~s}$ from C. atrox and C. adamanteus venoms, but only $78 \%$ identical to that of Cvv-E6f (Fig. 3).

Thus, the acid $\mathrm{PLA}_{2} \mathrm{~s}$ showed more geographic variations than the basic $\mathrm{PLA}_{2}$ in the venom of Cvv. In analogy, two or three acidic $\mathrm{PLA}_{2}$ isoforms have been found to be responsible for the geographic variations of the venoms of C. r. ruber [24] and Calloselasma rhodostoma [25]. In all the cases, the acidic $\mathrm{PLA}_{2} \mathrm{~S}$ show qualitative variations. This is probably attributable to more constrained or narrow functional specificities of the acidic $\mathrm{PLA}_{2} \mathrm{~s}$.

In addition to the present study, the cDNAs encoding hybrid-like $\mathrm{PLA}_{2} \mathrm{~S}$ were previously cloned from venom glands of Agkistrodon halys Pallas [26] and Ca. rhodostoma [15]. The presence of hybrid-like mRNAs encoding the venom serine proteases has been found in an Asian viper (our unpublished results). Notably, these clones appear to be different between individual snakes, i.e., those encoding PLAs-g and $-\mathrm{h}$ were derived from Cvv No.1 while the others (Fig. 4) were from Cvv No.2. The hybrid-like PLAs have never been expressed or purified in the venoms for all the cases so far studied. Therefore regulation mechanisms must exist to halt translation or secretion of the proteins in the venom glands.

To understand the mechanism behind the intraspecific venom variations, it is important to understand the structure-activity relationships and prey specificity of the venom proteins. Among the Cvv-E6 variants, the hot spots of mutations were found at residues 7-13, 20, 34, and 117-130 (Fig. 4), i.e., the surface residues located in the water-lipid interface of the enzyme [1,2]. Moreover, Cvv-E6a contains a unique "RGD motif" at 128-130, the motif for platelet binding through the platelet integrin GPIIb/IIIa [27,28]. In addition, all the Cvv-E6 PLA $_{2}$ s contain a proline bracket at the C-terminal region 122-131, suggesting possible functional importance of this region [28]. Notably, since Cvv-E6e and Cvv-E6b differ by only a substitution at position 34, H34 probably contributes to stronger anti-platelet activities of Cvv-E6e and Cvv-E6a, but not the Y34 in Cvv-E6b and the Q34 in Cvv-E6f (Fig. 5).

Crystallographic [5] and mutagenesis studies [29] of the acidic venom $\mathrm{PLA}_{2}$ of $A$. halys Pallas (i.e., Ahp) illustrated that residues E6, F20, W21, Y113, D115, and W119 probably are important for the anti-platelet ac-

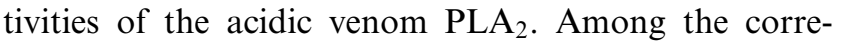
sponding sites in the Cvv-E6b, c, d, and f, only E6 and Y113 are conserved, while L20, S21, and I119 substitute for the aromatic residues (F20, W21, and W119 in the Ahp PLA $_{2}$ ) (Fig. 3). These substitutions possibly decrease the anti-platelet activities of Cvv-E6. On the other hand, the edema-inducing Lys49-PLA 2 s of pit viper venoms usually contained distinct substitutions such as R38, K78, D86, and K119 or R120 [30]. Interestingly, these substitutions were also found in Cvv-E6f, which showed lower enzymatic activity than the other Cvv-E6 isoforms, but significant edema-inducing activity not usually observed with other acidic PLA $2 \mathrm{~S}$ (Fig. 6).

It has been shown that the anti-platelet activities of venom $\mathrm{PLA}_{2} \mathrm{~s}$ depend on their enzymatic activities. The products of the enzymatic reaction, lysophospholipid and fatty acids, may inhibit signal pathways and alter the cytoskeleton of the platelet [31,32]. The enzymatic activities of Cvv-E6b, c, and d are relatively high but their anti-platelet activities are not potent. It remains to be studied whether some of the Cvv acidic $\mathrm{PLA}_{2}$ s mainly play a role in digestion of prey [33].

We have demonstrated [24] that an E6 PLA 2 of $\mathrm{Ca}$. rhodostoma venom, like Cvv-E6e, is a stronger inhibitor toward rabbit and rat platelets than another E6 PLA 2 of the same venom with stronger inhibition specificity for human platelets, like Cvv-E6a (Fig. 5). It is known that the platelets from different vertebrates may display rather distinct specificities toward exogeneous agents [34]. However, a parallel comparison of the diet composition and the venom $\mathrm{PLA}_{2}$ variants of each $\mathrm{Cvv}$ population is not possible at this time.

The venom proteins have been under natural selection for adaptation to ecological differences and the shift in diet composition [35-37]. The phylogenetic tree of the acidic $\mathrm{PLA}_{2} \mathrm{~S}$ (Fig. 7) also suggests the evolution of at least two subtypes of the venom E6 $\mathrm{PLA}_{2} \mathrm{~S}$ in the present-day C. v. viridis. Apparently, the multiple genes encoding different $\mathrm{PLA}_{2}$ variants may have resulted from gene duplication [17] and point mutations (e.g., among Cvv-E6b, E6c, and E6e). The quantitative differences in

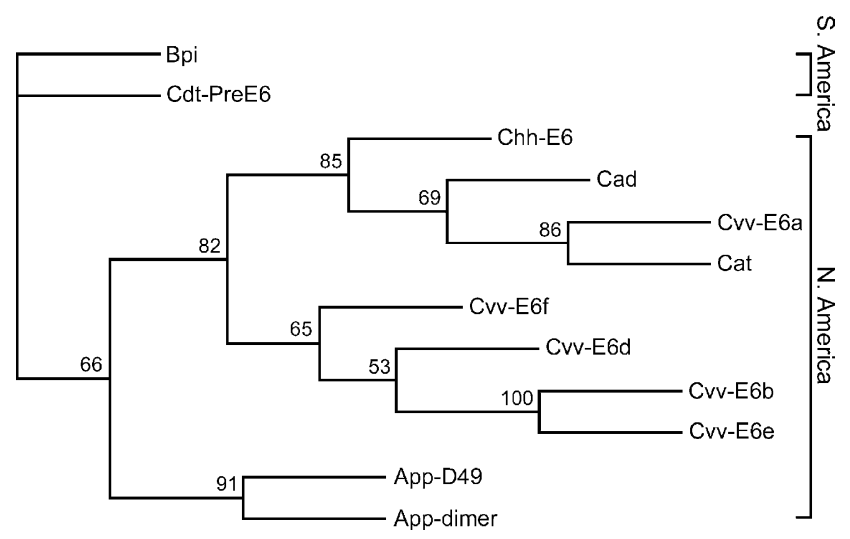

Fig. 7. Phylogenetic relationship between acidic venom $\mathrm{PLA}_{2}$ s from pit vipers of the New World. The amino acid sequences of the acidic venom $\mathrm{PLA}_{2} \mathrm{~S}$ were aligned and used as the dataset for phylogenetic analyses. The tree was constructed with the program PHYLIP with the E6-containing $\mathrm{PLA}_{2}$ from Bothrops pictus venom as the outgroup. The GenBank accession numbers for the $\mathrm{PLA}_{2} \mathrm{~S}$ and the species are Cvv6a-f of C. v. viridis(the present report); Chh-E6 of C. h. horridus (Tsai et al., unpublished result); Cad (P00623) of C. adamanteus; Cat (P00624) of C. atrox; App-D49 (P51972) and App-dimer (A53872) of A. p. piscivorus; Cdt-PreE6 (P08878) of C. d. terrificus; Bpi (AF288754) of $B$. pictus. 
the venom $\mathrm{PLA}_{2} \mathrm{~s}$ may have resulted from differential gene expression (e.g., among Cvv-N6, E6a, and E6f).

In conclusion, we demonstrated that the acidic $\mathrm{PLA}_{2} \mathrm{~S}$ in the $\mathrm{Cvv}$ geographic samples are relatively diversified, which possibly resulted from the necessary adaptation to great variations in the diet environment [34-36] and as a strategy to cope with platelet properties of different preys (Fig. 5). It is found for the first time that in addition to inhibiting platelet aggregation, the role of certain venom acidic $\mathrm{PLA}_{2} \mathrm{~s}$ (e.g., Cvv-E6f) may become edema induction, or even other less studied functions (e.g., digestion of the prey).

\section{Acknowledgments}

We heartily thank Professor E. Rael and Professor S.P. Mackessy for the generous gifts of C. v. viridis venom. This study has been supported by research grants from the Administration of Education (Grant 89-BFA01-1-4) and National Science Council of Taiwan, ROC.

\section{References}

[1] J.M. Danse, S. Gasparini, A. Menez, in: R.M. Kini (Ed.), Venom Phospholipase $\mathrm{A}_{2}$ Enzyme: Structure, Function and Mechanism, Wiley, UK, 1997, pp. 29-71.

[2] B.Z. Yu, J. Rogers, M.D. Tsai, C. Pidgeon, M.K. Jain, Biochemistry 38 (1999) 4875-4884.

[3] I.-H. Tsai, J. Toxicol. Toxin Rev. 16 (1997) 79-114.

[4] B.R. Francis, J. Meng, I.I. Kaiser, in: G.S. Bailey (Ed.), Enzymes from Snake Venom, Alaken Inc., Colorado, USA, 1998, pp. 503544.

[5] X.-Q. Wang, J. Yang, L.-L. Gui, Z.-J. Lin, Y.-C. Chen, Y.-C. Zhou, J. Mol. Biol. 255 (1996) 669-676.

[6] R.M. Kini, H.J. Evans, in: R.M. Kini (Ed.), Venom Phoshpholipase $\mathrm{A}_{2}$ Enzyme: Structure, Function and Mechanism, Wiley, UK, 1997, pp. 369-387.

[7] J.L. Glenn, R.C. Straight, in: A.T. Tu (Ed.), Rattlesnake Venoms: Their Actions and Treatments, Marcel Dekker, New York, 1982, pp. 3-120.

[8] Y. Komori, T. Nikai, H. Sugihara, Biochim. Biophys. Acta 967 (1988) 92-102.

[9] A.L. Bieber, D. Nedelkov, J. Toxicol. Toxin Rev. 16 (1997) 33-52.

[10] C.L. Ownby, T.R. Colberg, S.P. White, Toxicon 35 (1997) 111 124.
[11] M. Anaya, E.D. Rael, C.S. Lieb, J.C. Perez, R.J. Salo, J. Herpetol. 26 (1992) 473-482.

[12] C.E. Pook, W. Wuster, R.S. Thorpe, Mol. Phylogenet. Evol. 15 (2000) 269-282.

[13] I.H. Tsai, P.J. Lu, Y.M. Wang, C.L. Ho, L.L. Liaw, Biochem. J. 311 (1995) 895-900.

[14] J.W. Norris, R.M. Fry, A.T. Tu, Biochem. Biophys. Res. Commun. 230 (1997) 607-610.

[15] I.H. Tsai, Y.M. Wang, L.C. Au, T.P. Ko, Y.H. Chen, Y.F. Chu, Eur. J. Biochem. 267 (2000) 6684-6691.

[16] K.B. Mullis, F. Faloona, Methods Enzymol. 155 (1987) 335-350.

[17] T. Ogawa, M. Kitajima, K.I. Nakashima, Y. Sakaki, M. Ohno, J. Mol. Evol. 41 (1995) 867-877.

[18] T. Maniatis, E.F. Fritsch, J. Sambrook, Molecular Cloning: A Laboratory Manual, Cold Spring Harbor Laboratory, Cold Spring Harbor, NY, 1989.

[19] J. Felsenstein, PHYLIP: the PHYLogeny Inference Package, version 3.573. Computer program distributed by the University of Washington, Department of Genetics, Seattle, 1992.

[20] J. Felsenstein, Evolution 39 (1985) 783-791.

[21] R. Renetseder, B.W. Dijkstra, K. Huizinga, K.H. Kalk, J. Drenth, J. Mol. Biol. 200 (1988) 181-188.

[22] R.M. Scarborough, J.W. Rose, M.A. Naughton, D.R. Phillips, L. Nannizzi, A. Arfsten, A.M. Campbell, I.F. Charo, J. Biol. Chem. 268 (1993) 1058-1065.

[23] K.G. Ashton, A. de Queiroz, Mol. Phylogenet. Evol. 21 (2001) 176-189.

[24] R.C. Straight, J.L. Glenn, T.B. Wolt, M.C. Wolfe, Comp. Biochem. Physiol. 103B (1992) 635-639.

[25] I.H. Tsai, Y.H. Chen, Y.M. Wang, Arch. Biochem. Biophys. 387 (2001) 257-264.

[26] H. Pan, X.-L. Liu, L.-L. Ou-Yang, G.-Z. Yang, Y.-C. Zhou, Z.-P. Li, X.-F. Wu, Toxicon 36 (1998) 1155-1163.

[27] T.F. Huang, S. Niewiarowski, J. Toxicol. Toxin Rev. 13 (1994) 253-271.

[28] R.M. Kini, H.J. Evan, FEBS Lett. 375 (1995) 15-17.

[29] X.L. Liu, X.F. Wu, Y.C. Zhou, J. Nat. Toxins 10 (2001) 43-55.

[30] I.H. Tsai, Y.H. Chen, Y.M. Wang, M.C. Tu, A.T. Tu, Arch. Biochem. Biophys. 394 (2001) 236-244.

[31] Y. Yuan, S.P. Jackson, C.A. Mitchell, H.H. Salem, Thromb. Res. 70 (1993) 471-481.

[32] Y. Yuan, S.M. Schoenwaelder, H.H. Salem, S.P. Jackson, J. Biol. Chem. 271 (1996) 27090-27098.

[33] R.G. Thomas, F.H. Pough, Toxicon 17 (1979) 221-228.

[34] Y.L. Chen, T.F. Huang, S.W. Chen, I.H. Tsai, Biochem. J. 305 (1995) 513-520.

[35] S. Creer, W.H. Chou, A. Malhotra, R.S. Thorpe, Zool. Sci. 19 (2002) 907-913.

[36] S. Creer, A. Malhotra, R.S. Thorpe, W.H. Chou, Mol. Ecol. 10 (2001) 1967-1981.

[37] J.C. Daltry, W. Wüster, R.S. Thorpe, J. Herpetol. 32 (1998) 198 205. 